\title{
A Generalization OF \\ THE BARONE-ADESI AND \\ WHALEY APPROACH FOR THE \\ ANALYTIC APPROXIMATION OF \\ AMERICAN OPTIONS
}

\author{
JIA-HAU GUO \\ MAO-WEI HUNG* \\ LEH-CHYAN SO
}

This article introduces a general quadratic approximation scheme for pricing American options based on stochastic volatility and double jump processes. This quadratic approximation scheme is a generalization of the Barone-Adesi and Whaley approach and nests several option models. Numerical results show that this quadratic approximation scheme is efficient and useful in pricing American options. (C) 2009 Wiley Periodicals, Inc. Jrl Fut Mark 29:478-493, 2009

\footnotetext{
The authors thank an anonymous referee for useful comments.

*Correspondence author, College of Management, National Taiwan University, No. 1, Section 4, Roosevelt Road, Taipei, Taiwan. Tel: 886-2-3366-4988, Fax: 886-2-2369-0833, e-mail: hung@management.ntu.edu.tw Accepted 12 May 2008
}

- Jia-Hau Guo is from the College of Management, National Chiao Tung University, Hsinchu, Taiwan.

- Mao-Wei Hung is from the College of Management, National Taiwan University, Taipei, Taiwan.

- Leh-Chyan So is from the College of Technology Management, National Tsing Hua University, Hsinchu, Taiwan. 


\section{INTRODUCTION}

The goal of this research is to provide an efficient analytic approximation for pricing American options in a general model that allows for stochastic volatility, return jumps, and volatility jumps. The fact that asset returns exhibit both stochastic volatility and jumps has been widely documented. Although some closed-form solutions for European options based on these diffusion processes have been derived in recent years, no known analytic solution for American options exists. Consequently, simulative and numerical approaches are used to calculate American option values.

Barone-Adesi and Whaley (1987) originally applied the quadratic approximation method to price American options using the decomposition technique. Bates (1991) first extended this method by introducing jumps into the process of the underlying asset return. Ju and Zhong (1999) improved the accuracy of the original method for options with intermediate maturities by adding a correction term onto the approximation. Chang, Kang, Kim, and Kim (2007) introduced an additional parameter into the original method and succeeded in extending its application from basic options to barrier options and lookback options. Nevertheless, these methods do not continuously evolve in conjunction with the rapid growth of new option pricing models in the stochastic volatility framework. Additional empirical studies provide evidence that an option model allowing for stochastic volatility and double jumps dramatically reduces option-pricing errors (see Bakshi, Cao, \& Chen, 1997; Broadie, Chernov, \& Johannes, 2007; and others). These studies illustrate the importance of extending the quadratic approximation method for American options based on these processes.

Stochastic volatility models with double jumps proposed by Duffie, Pan, and Singleton (2000) were used as examples to highlight the generalization of the authors' quadratic approximation scheme, followed by a comparison with the least-squares simulation approach proposed by Longstaff and Schwartz (2001). The results of the authors' comparison show that the quadratic approximation scheme is useful and efficient in pricing American options based on these diffusion processes. To illustrate its generality, this approximation scheme is also applied to other existing models.

The remainder of this article is organized as follows: the second section briefly describes the stochastic volatility model with correlated double jumps and the authors' quadratic approximation scheme. The third section gives analytic approximation formulae for other jump models. The fourth section compares quadratic approximations with the least-squares simulation approach. Conclusions are presented in the fifth section. 


\section{ANALYTIC AMERICAN OPTION APPROXIMATIONS FOR THE STOCHASTIC VOLATILITY MODEL WITH DOUBLE JUMPS}

The primary expression of the stochastic volatility model with double jumps, adopted from Duffie et al. (2000) was reproduced to generate a self-contained treatment. Under the risk-neutral measure, the underlying asset price, $S(t)$, is posited to follow a geometric jump diffusion with the instantaneous conditional variance, $Y(t)$, following a mean-reverting square root jump process:

$$
\begin{gathered}
\frac{d S(t)}{S(t)}=(r-d) d t+\sqrt{Y(t)} d W_{S}(t)+\left(e^{x(t)}-1\right) d q_{S}(t)-E^{Q}\left[\left(e^{x(t)}-1\right) d q_{S}(t)\right] \\
d Y(t)=\left(\bar{Y}-\kappa_{Y} Y(t)\right) d t+\sigma_{Y} \sqrt{Y(t)} d W_{Y}(t)+y(t) d q_{Y}(t), \quad t \geq 0
\end{gathered}
$$

where $r$ is the risk-free interest rate and $d$ is the dividend yield. $x(t)$ represents a percentage jump in the stock price and follows a normal distribution, $N\left(\mu_{0}+\mu_{x, y} y, \sigma_{x, y}^{2}\right)$, where $y(t)$ is a level jump in the volatility and follows an exponential distribution, Exponential $\left(\theta_{y}\right) \cdot q_{S}(t)$ and $q_{Y}(t)$ are two correlated Poisson counters with intensity $\lambda_{x, y} \cdot \rho$ denotes the instantaneous correlation coefficient between the stock price return process and its conditional variance process. To retain the Martingale property, the compensator, $E^{Q}\left[(\exp [x(t)]-1) d q_{S}(t)\right]=$ $\lambda_{x, y}\left(\exp \left[\mu_{0}+0.5 \sigma_{x, y}^{2}\right] /\left(1-\theta_{y} \mu_{x, y}\right)-1\right) d t$, is subtracted from the stock price process, such that the drift of the stock return rate is equal to $r-d$.

The partial integro-differential equation for a contingent claim price, $P$, on the underlying asset is given by

$$
\begin{aligned}
0= & \frac{1}{2} P_{S S} S^{2} Y+\frac{1}{2} P_{Y Y} \sigma_{Y}^{2} Y+P_{S Y} \rho \sigma_{Y} S Y \\
& +P_{S}\left((r-d)-\lambda^{x, y} E^{Q}\left[e^{x}-1\right]\right) S+P_{Y}\left(\bar{Y}-\kappa_{Y} Y\right)-P_{T}-r P \\
& +\lambda^{x, y} \int_{0}^{\infty} \int_{-\infty}^{\infty}\left[P\left(S e^{x}, Y+y\right)-P(S, Y)\right] \Phi(x, y) d x d y .
\end{aligned}
$$

Compared with European option values, American option values can be exercised at any time before maturity. The flexibility of the right-to-exercise options determines the "early-exercise premium" markup of American prices over European option prices. MacMillan (1987), Barone-Adesi and Whaley (1987), and Bates (1991) decomposed the value of an American option into its European counterpart and an early-exercise premium to obtain an approximation formula. The price of a basic American call option, $C^{A}(S, Y, T ; K)$, with a strike price, $K$, and a maturity date, $T$, can be represented as 


$$
C^{A}(S, Y, T ; K)=C^{E}(S, Y, T ; K)+\varsigma(S, Y, T ; K)
$$

where $C^{E}(S, Y, T ; K)$ is the price of the corresponding European call option and $\varsigma(S, Y, T ; K)$ is the value of the corresponding early-exercise premium. Given the linearity of Equation (4), the early-exercise premium must satisfy Equation (3) because American option values, as well as European option values, satisfy the aforementioned partial differential equation in the nonstopping region under the risk-neutral measure.

Given an analytic European option solution ${ }^{1}$ (see Appendix A), the only unsolved problem in deriving the pricing formula of an American option is a good approximation for the early-exercise premium. Because options are homogeneous in $S$ and $K$, the premium is also homogeneous in $S$ and $K$ :

$\varsigma(S, Y, T ; K)=K s(S / K, Y, T ; 1)$. The Barone-Adesi and Whaley method (1987) was used to define the premium as

$$
\varsigma(S, Y, T ; K)=K H(T) F(S / K, Y, H)=K H(T) F(z, Y, H)
$$

where $z \equiv S / K$ and $H(T)$ is an arbitrary function of time-to-maturity, T. The partial derivatives of $s$ are $\varsigma_{S}=H F_{z}, \varsigma_{S S}=H F_{z z} / K, \varsigma_{Y}=K H F_{Y}, \varsigma_{Y Y}=K H F_{Y Y}, \varsigma_{S Y}=$ $H F_{z Y}$, and $s_{T}=K F H_{T}=K H F_{H} H_{T}$. Equation (6) is generated by substituting Equation (5) into Equation (3)

$$
\begin{aligned}
0= & \frac{1}{2} K H F_{z z^{2}} z^{2}+K H F_{z}\left((r-d)-\lambda^{x, y} E\left[e^{x}-1\right]\right) z+\frac{1}{2} K H F_{Y Y} \sigma_{Y}^{2} Y \\
& +K H F_{z Y} \rho \sigma_{Y} z Y+K H F_{Y}\left(\bar{Y}-\kappa_{Y} Y\right)-H_{T}\left(K F+K H F_{H}\right) \\
& -r K H F+\lambda^{x, y} K H \int_{0}^{\infty} \int_{-\infty}^{\infty}\left[F\left(z e^{x}, Y+y\right)-F(z, Y)\right] \Phi(x, y) d x d y .
\end{aligned}
$$

Barone-Adesi and Whaley (1987) chose $H(T)$ as $1-\exp [-r T]$ for simplicity. Chang et al. (2007) further adjusted $H(T)$ to equal $1-\exp [-\alpha r T]$ for controlling $\alpha$ to reduce barrier option pricing errors of the quadratic approximation. After substituting $H(T)=1-\exp [-\alpha \mathrm{rTT}]$ into Equation (6), Equation (7) resulted:

$$
\begin{aligned}
0= & \frac{1}{2} K H F_{z z} z^{2} Y+K H F_{z}\left((r-d)-\lambda^{x, y} E\left[e^{x}-1\right]\right) z+\frac{1}{2} K H F_{Y Y} \sigma_{Y}^{2} Y \\
& +K H F_{z Y} \rho \sigma_{Y} z Y+K H F_{Y}\left(\bar{Y}-\kappa_{Y} Y\right)-\alpha r(1-H) K F-\alpha r(1-H) K H F_{H} \\
& -r K H F+\lambda^{x, y} K H \int_{0}^{\infty} \int_{-\infty}^{\infty}\left[F\left(z e^{x}, Y+y\right)-F(z, y)\right] \Phi(x, y) d x d y .
\end{aligned}
$$

${ }^{1}$ Guo and Hung (2007) suggested a simple way to avoid the branch cut difficulties arising from the choice of the branch of the complex logarithm in the implementation of the European option solution. 
As described in Barone-Adesi and Whaley (1987), Bates (1991), and Chang et al. (2007), $\alpha r[1-H] K H F_{H}$ is negligible. Substituting $F(z, Y)=$ $A_{1} \exp \left[B_{1} Y\right] z^{\phi_{1}}+A_{2} \exp \left[B_{2} Y\right] z^{\phi_{2}}$ into Equation (7) and separating variables $A_{1}$ and $A_{2}$, yields

$$
\begin{aligned}
0= & \frac{1}{2} \phi(\phi-1) Y+\phi\left((r-d)-\lambda^{x, y}\left(\frac{\exp \left[\mu_{0}+\frac{1}{2} \sigma_{x, y}^{2}\right]}{1-\theta_{y} \mu_{x, y}}-1\right)\right) \\
& +\frac{1}{2} B^{2} \sigma_{Y}^{2} Y+\phi B \rho \sigma_{Y} Y+B\left(\bar{Y}-\kappa_{Y} Y\right)-\alpha r\left(\frac{1}{H}-1\right)-r \\
& +\lambda^{x, y}\left(\frac{\exp \left[\frac{1}{2} \phi^{2} \sigma_{x, y}^{2}+\mu_{0} \phi\right]}{1-B \theta_{y}-\phi \mu_{x, y} \theta_{y}}-1\right) .
\end{aligned}
$$

After further separating Equation (8) into two equations for Y-terms and non-Y-terms, respectively, Equations (9) and (10) are generated.

$$
0=\frac{1}{2} \phi^{2}+\left(B \rho \sigma_{Y}-\frac{1}{2}\right) \phi+\frac{1}{2} B^{2} \sigma_{Y}^{2}-B \kappa_{Y}
$$

and

$$
\begin{aligned}
0= & B \bar{Y}-\left(\alpha r\left(\frac{1}{H}-1\right)+r\right)+\left((r-d)-\lambda^{x, y}\left(\frac{\exp \left[\mu_{0}+\frac{1}{2} \sigma_{x, y}^{2}\right]}{1-\theta_{y} \mu_{x, y}}-1\right)\right) \phi \\
& +\lambda^{x, y}\left(\frac{\exp \left[\frac{1}{2} \phi^{2} \sigma_{x, y}^{2}+\mu_{0} \phi\right]}{1-B \theta_{y}-\phi \mu_{x, y} \theta_{y}}-1\right) .
\end{aligned}
$$

For given values of the parameters $r, d, \bar{Y}, \kappa_{Y}, \sigma_{Y}, \rho, \lambda^{x, y}, \mu_{0}, \mu_{x, y}, \sigma_{x, y}, \theta_{y}$, and $\alpha$, accurate values of $\phi_{1}, \phi_{2}, B_{1}$, and $B_{2}$ can be rapidly determined from Equations (9) and (10) using Newton's method. Initial values are obtained from Equation (10) given by replacing $\exp \left[\left(-\left(B+\phi \mu_{x, y}\right) \theta_{y}\right)\right] \cong 1-B \theta_{y}-$ $\phi \mu_{x, y} \theta_{y}$ and expanding $\exp \left[\left(\mu_{0}+\mu_{x, y} \theta_{y}\right) \phi+\sigma_{x, y}^{2} \phi^{2} / 2+\theta_{y} B\right]$ in a first-order Taylor expansion, ignoring powers of $\phi$ and $B$ higher than 2 . The result of the approximation of Equation (10) is

$$
B=\left(\eta_{0}-\eta_{1} \phi\right) /\left(\bar{Y}+\lambda^{x, y} \theta_{y}\right)
$$

where $\eta_{0}=\alpha r(1-H) / H+r$ and $\eta_{1}=(r-d)+\lambda^{x, y}\left(\mu_{0}+\mu_{x, y} \theta_{y}\right)$ $-\lambda^{x, y}\left(\exp \left[\mu_{0}+\sigma_{x, y}^{2} / 2\right] /\left(1-\theta_{y} \mu_{x, y}\right)-1\right)$. Equation (11) indicates that for 
jumps with plausible amplitudes $\left(\left|\mu_{0}\right|,\left|\mu_{x, y}\right|, \sigma_{x, y}\right.$ and $\theta_{y}$ substantially less than 1), a one-to-one relationship exists between $\phi$ and B. Substituting Equation (11) into Equation (9) generates

$$
\begin{aligned}
0= & \left(\left(\bar{Y}+\lambda^{x, y} \theta_{y}\right)^{2}+\sigma_{Y}^{2} \eta_{1}^{2}-2 \rho \sigma_{Y}\left(\bar{Y}+\lambda^{x, y} \theta_{y}\right) \eta_{1}\right) \phi^{2} \\
& +\left(2 \rho \sigma_{Y}\left(\bar{Y}+\lambda^{x, y} \theta_{y}\right) \eta_{0}+2 \kappa_{Y}\left(\bar{Y}+\lambda^{x, y} \theta_{y}\right) \eta_{1}-\left(\bar{Y}+\lambda^{x, y} \theta_{y}\right)^{2}-2 \sigma_{Y}^{2} \eta_{0} \eta_{1}\right) \phi \\
& +\left(\sigma_{Y}^{2} \eta_{0}^{2}-2 \kappa_{Y}\left(\bar{Y}+\lambda^{x, y} \theta_{y}\right) \eta_{0}\right) .
\end{aligned}
$$

Parameters that satisfy the relationship $\eta_{0}<2 \kappa_{Y}\left(\bar{Y}+\lambda^{x, y} \theta_{y}\right) / \sigma_{Y}^{2}$ guarantee that one root $\left(\phi_{1}\right)$ is negative for puts, whereas the other $\left(\phi_{2}\right)$ is positive for calls. Because the relationships $\phi_{1}<0$ and $A_{1} \neq 0$ imply that the $\lim _{\mathrm{S} \rightarrow 0} C^{A}$ $(S, Y ; T, K)=\infty$, therefore, it follows that $A_{1}=0$. Once values for $\phi_{2}$ and $B_{2}$ are obtained, $A_{2}$ and $\bar{S}$ (the critical early-exercise price for calls) can be solved from the value-match condition and the high contact condition:

$$
\begin{aligned}
\varsigma(\bar{S}, Y, T ; K) & =\bar{S}-K-C^{E}(\bar{S}, Y, T ; K) \text { and } \\
\varsigma_{S}(\bar{S}, Y, T ; K) & =1-C_{S}^{E}(\bar{S}, Y, T ; K) .
\end{aligned}
$$

Equations (13) and (14) imply that $\bar{S}$ is the implicit solution to

$$
\bar{S}=\frac{\phi_{2}\left(\bar{S}-K-C^{E}(\bar{S}, Y, T ; K)\right)}{1-C_{z}^{E}(\bar{S}, Y, T ; K)}
$$

and $A_{2}$ can be determined by

$$
A_{2}=\frac{\bar{S}-K-C^{E}(\bar{S}, Y, T ; K)}{K(1-\exp [-\alpha r T]) \exp \left[B_{2} Y\right]\left(\frac{\bar{S}}{K}\right)^{\phi_{2}}}
$$

The resulting formula for a basic American call option is

$$
\begin{gathered}
C^{A}(S, Y, T ; K)=C^{E}(S, Y, T ; K)+\left(\bar{S}-K-C^{E}(\bar{S}, Y, T ; K)\right)(S / \bar{S})^{\phi_{2}} \\
\text { for } S<\bar{S} \text { or } \bar{S}-K \text { for } S \geq \bar{S} .
\end{gathered}
$$

Although the American puts must satisfy the same partial differential equation, the boundary conditions are somewhat different compared with those for calls. The boundary conditions for puts are

and

$$
P^{A}(\underline{S}, Y, T ; K)=K-\underline{S}
$$

$$
P_{S}^{A}(\underline{S}, Y, T ; K)=-1
$$


where $\underline{S}$ is the critical early-exercise price. The positive root $\left(\phi_{2}\right)$ is precluded for the puts because it implies that the $\lim _{S \rightarrow \infty} P^{A}(S, Y ; T, K)=\infty$. Consequently, $A_{2}=0$ and therefore,

$$
\begin{aligned}
P^{A}(S, Y, T ; K)= & P^{E}(S, Y, T ; K)+\left(K-\underline{S}-P^{E}(\underline{S}, Y, T ; K)\right)(S / \underline{S})^{\phi_{1}} \\
& \text { for } S>\underline{S} \text { or } K-\underline{S} \text { for } S \leq(\underline{S})
\end{aligned}
$$

where

$$
\underline{S}=\frac{\phi_{1}\left(K-\underline{S}-P^{E}(\underline{S}, Y, T ; K)\right)}{-1-P_{S}^{E}(\underline{S}, Y, T ; K)} .
$$

Note that, after turning off the specification of stochastic volatility and volatility jumps $\left(\kappa_{Y} Y=\bar{Y}, \rho=0, \sigma_{Y}=0, \mu_{x, y}=0\right.$, and $\left.\theta_{y}=0\right)$, the authors' general solution reduces to that of Bates (1991). Table I demonstrates that their results are consistent with the results in this case. Data in Table I for Bates and for the finite difference method are taken from Table II in Bates (1991).

\begin{tabular}{|c|c|c|c|c|c|c|c|}
\hline & $K$ & $\sigma_{Y}=0.5$ & $\sigma_{Y}=0.25$ & $\sigma_{Y}=0.1$ & $\sigma_{Y}=0.01$ & Bates & $F D$ \\
\hline \multirow[t]{5}{*}{$P$} & 220 & $\begin{array}{c}0.223 \\
(0.2196)\end{array}$ & $\begin{array}{l}0.209 \\
(0.2058)\end{array}$ & $\begin{array}{c}0.196 \\
(0.1923)\end{array}$ & $\begin{array}{l}0.19 \\
(0.1866)\end{array}$ & $\begin{array}{c}0.19 \\
\mathbf{( 0 . 1 9 )}\end{array}$ & $\begin{array}{c}0.19 \\
\mathbf{( 0 . 1 9 )}\end{array}$ \\
\hline & 235 & $\begin{array}{c}1.612 \\
(1.6007)\end{array}$ & $\begin{array}{c}1.621 \\
(1.6091)\end{array}$ & $\begin{array}{c}1.628 \\
(1.6159)\end{array}$ & $\begin{array}{c}1.631 \\
(1.6186)\end{array}$ & $\begin{array}{c}1.63 \\
\mathbf{( 1 . 6 2 )}\end{array}$ & $\begin{array}{c}1.63 \\
(1.62)\end{array}$ \\
\hline & 250 & $\begin{array}{c}6.773 \\
(6.7330)\end{array}$ & $\begin{array}{l}6.807 \\
(6.7664)\end{array}$ & $\begin{array}{c}6.839 \\
(6.798)\end{array}$ & $\begin{array}{c}6.852 \\
(6.8109)\end{array}$ & $\begin{array}{c}6.85 \\
(6.81)\end{array}$ & $\begin{array}{c}6.82 \\
(6.81)\end{array}$ \\
\hline & 265 & $\begin{array}{c}16.918 \\
(16.7916)\end{array}$ & $\begin{array}{c}16.923 \\
(16.7942)\end{array}$ & $\begin{array}{c}16.925 \\
(16.7949)\end{array}$ & $\begin{array}{c}16.926 \\
(16.7949)\end{array}$ & $\begin{array}{c}16.91 \\
(16.79)\end{array}$ & $\begin{array}{c}16.90 \\
(16.79)\end{array}$ \\
\hline & 280 & $\begin{array}{c}30.223 \\
(29.8443)\end{array}$ & $\begin{array}{c}30.217 \\
(29.8325)\end{array}$ & $\begin{array}{c}30.210 \\
(29.821)\end{array}$ & $\begin{array}{c}30.208 \\
(29.8162)\end{array}$ & $\begin{array}{c}30.19 \\
(29.82)\end{array}$ & $\begin{array}{c}30.21 \\
(29.82)\end{array}$ \\
\hline \multirow[t]{5}{*}{ C } & 220 & $\begin{array}{c}30.014 \\
(29.4789)\end{array}$ & $\begin{array}{l}30.011 \\
(29.4651)\end{array}$ & $\begin{array}{c}30.009 \\
(29.4516)\end{array}$ & $\begin{array}{c}30.008 \\
(29.4459)\end{array}$ & $\begin{array}{c}30.01 \\
(29.45)\end{array}$ & $\begin{array}{c}30.00 \\
(29.45)\end{array}$ \\
\hline & 235 & $\begin{array}{c}16.398 \\
(16.2304)\end{array}$ & $\begin{array}{c}16.410 \\
(16.2387)\end{array}$ & $\begin{array}{c}16.420 \\
(16.2456)\end{array}$ & $\begin{array}{c}16.425 \\
(16.2483)\end{array}$ & $\begin{array}{c}16.42 \\
(16.25)\end{array}$ & $\begin{array}{c}16.41 \\
(16.25)\end{array}$ \\
\hline & 250 & $\begin{array}{c}6.789 \\
(6.733)\end{array}$ & $\begin{array}{c}6.824 \\
(6.7664)\end{array}$ & $\begin{array}{c}6.857 \\
(6.798)\end{array}$ & $\begin{array}{c}6.870 \\
(6.8109)\end{array}$ & $\begin{array}{c}6.86 \\
(6.81)\end{array}$ & $\begin{array}{c}6.84 \\
(6.81)\end{array}$ \\
\hline & 265 & $\begin{array}{l}2.182 \\
(2.162)\end{array}$ & $\begin{array}{c}2.185 \\
(2.1645)\end{array}$ & $\begin{array}{l}2.186 \\
(2.1653)\end{array}$ & $\begin{array}{c}2.187 \\
(2.1653)\end{array}$ & $\begin{array}{c}2.18 \\
(2.17)\end{array}$ & $\begin{array}{l}2.18 \\
(2.17)\end{array}$ \\
\hline & 280 & $\begin{array}{c}0.593 \\
(0.585)\end{array}$ & $\begin{array}{c}0.581 \\
(0.5732)\end{array}$ & $\begin{array}{c}0.570 \\
(0.5617)\end{array}$ & $\begin{array}{c}0.565 \\
(0.5569)\end{array}$ & $\begin{array}{c}0.56 \\
(0.56)\end{array}$ & $\begin{array}{c}0.56 \\
(0.56)\end{array}$ \\
\hline
\end{tabular}

TABLE I

American Option Values Under Stochastic Volatility When $\sigma_{Y} \rightarrow 0$

Note. $S=250, T=0.25, r=0.1, d=0.1, Y=0.01, \lambda^{x, y}=10, \mu_{0}=0.01005, \sigma_{x, y}=0.03, \rho=0, \mu_{x, y}=0 \theta_{y}=0, \kappa_{Y} Y=$ $\bar{Y}=0.24 \sigma_{Y}$, and $\alpha=1$. Puts (P) and Calls (C), the Bates' approximation at $\sigma_{Y}=0$ (Bates), and the finite difference method (FD) are abbreviated accordingly. European option values are given in parentheses. 
TABLE II

Comparisons of American Options: Correlated Double Jumps

\begin{tabular}{|c|c|c|c|c|c|c|}
\hline & $S$ & European Option & Simulated American & (s.e.) & Approx & Diff \\
\hline \multirow[t]{7}{*}{$P$} & 60 & 39.501 & 40.006 & 0.026 & 40.000 & 0.005 \\
\hline & 70 & 29.936 & 30.173 & 0.038 & 30.138 & 0.035 \\
\hline & 80 & 20.948 & 21.067 & 0.034 & 21.033 & 0.034 \\
\hline & 90 & 13.302 & 13.365 & 0.044 & 13.341 & 0.024 \\
\hline & 100 & 7.786 & 7.843 & 0.048 & 7.805 & 0.038 \\
\hline & 110 & 4.417 & 4.463 & 0.049 & 4.428 & 0.036 \\
\hline & 120 & 2.546 & 2.575 & 0.047 & 2.552 & 0.023 \\
\hline \multirow[t]{7}{*}{ C } & 60 & 0.097 & 0.106 & 0.011 & 0.097 & 0.009 \\
\hline & 70 & 0.382 & 0.423 & 0.028 & 0.383 & 0.040 \\
\hline & 80 & 1.246 & 1.287 & 0.033 & 1.247 & 0.040 \\
\hline & 90 & 3.451 & 3.517 & 0.053 & 3.454 & 0.062 \\
\hline & 100 & 7.786 & 7.858 & 0.079 & 7.794 & 0.064 \\
\hline & 110 & 14.268 & 14.351 & 0.069 & 14.286 & 0.064 \\
\hline & 120 & 22.249 & 22.362 & 0.051 & 22.288 & 0.073 \\
\hline
\end{tabular}

Note. $K=100, T=0.25, r=0.06, d=0.06, \bar{Y}=0.49, Y=0.0968, \rho=-0.1, \lambda^{x, y}=1.64, \mu_{0}=-0.03, \mu_{x, y}=-7.87$, $\sigma_{x, y}=0.22, \theta_{y}=0.0036, \sigma_{Y}=0.61, \kappa_{Y}=5.06$, and $\alpha=1$.

\section{ANALYTIC APPROXIMATION FORMULAE FOR OTHER JUMP MODELS}

The quadratic approximation scheme can be applied to the stochastic volatility model with other jump types, such as independent return jumps and volatility jumps. The partial integro-differential equation for a contingent claim price, $P$, on the underlying asset is given by

$$
\begin{aligned}
0= & \frac{1}{2} P_{S S} S^{2} Y+\frac{1}{2} P_{Y Y} \sigma_{Y}^{2} Y+P_{S Y} \rho \sigma_{Y} S Y \\
& +P_{S}\left((r-d)-\lambda E^{Q}\left[e^{x}-1\right]\right) S+P_{Y}\left(\bar{Y}-\kappa_{Y} Y\right)-P_{T}-r P \\
& +\lambda^{x} \int_{-\infty}^{\infty}\left[P\left(S e^{x}\right)-P(S)\right] \Phi(x) d x+\lambda^{y} \int_{0}^{\infty}[P(Y+y)-P(Y)] \Phi(y) d y
\end{aligned}
$$

where $x$ is a return-jump amplitude, $\lambda^{x}$ is the arrival rate of return jumps, $y$ is a volatility-jump amplitude, and $\lambda^{y}$ is the arrival rate of volatility jumps.

It is assumed that $x$ follows a normal distribution, $N\left(\log \left(1+\mu_{x}\right)-\frac{1}{2} \sigma_{x}^{2}, \sigma_{x}^{2}\right)$, and $y$ follows an exponential distribution, Exponential $\left(\theta_{y}\right)$. Equation (22) induces the following differential equation: 


$$
\begin{aligned}
0= & \frac{1}{2} \phi(\phi-1) Y+\phi\left((r-d)-\lambda^{x} \mu_{x}\right)+\frac{1}{2} B^{2} \sigma_{Y}^{2} Y+\phi B \rho \sigma_{Y} Y+B\left(\bar{Y}-\kappa_{Y} Y\right) \\
& -\alpha r\left(\frac{1}{H}-1\right)-r+\lambda^{x}\left(\exp \left[\phi \log \left[1+\mu_{x}\right]+\frac{1}{2} \sigma_{x}^{2} \phi(\phi-1)\right]-1\right) \\
& +\lambda^{y}\left(\frac{1}{1-\theta_{y} B}-1\right) .
\end{aligned}
$$

Further separating Equation (23) into two equations for Y-terms and nonY-terms, respectively, yields

$$
0=\frac{1}{2} \phi(\phi-1)+\frac{1}{2} B^{2} \sigma_{Y}^{2}+\phi B \rho \sigma_{Y}-B \kappa_{Y}
$$

and

$$
\begin{aligned}
0= & B \bar{Y}-\left(\operatorname{ar}\left(\frac{1}{H}-1\right)+r\right)+\left((r-d)-\lambda^{x} \mu_{x}\right) \phi \\
& +\lambda^{x}\left(\exp \left[\phi \log \left[1+\mu_{x}\right]+\frac{1}{2} \sigma_{x}^{2} \phi(\phi-1)\right]-1\right) \\
& +\lambda^{y}\left(\frac{1}{1-\theta_{y} B}-1\right) .
\end{aligned}
$$

Similarly, accurate values of $\phi_{1}, \phi_{2}, B_{1}$, and $B_{2}$ can be rapidly determined from Equations (24) and (25) using Newton's method. Initial values are obtained from Equation (25) given by replacing $1 /\left(1-\theta_{y} B\right)-1 \cong \theta_{y} B$ and expanding $\exp \left[\phi \log \left[1+\mu_{x}\right]+\sigma_{x}^{2} \phi(\phi-1) / 2\right]$ in a first-order Taylor expansion, ignoring powers of $\phi$ higher than 2. The approximation of Equation (25) results in Equation (26)

$$
B=\left(\eta_{0}-\eta_{1}^{*} \phi\right) /\left(\bar{Y}+\lambda^{y} \theta_{y}\right)
$$

where $\eta_{0}=\alpha r(1-H) / H+r$ and $\eta_{1}^{*}=(r-d)-\lambda^{x} \mu_{x}+\lambda^{x}\left(\log \left[1+\mu_{x}\right]-\frac{1}{2} \sigma_{x}^{2}\right.$. Substituting Equation (26) into Equation (24) yields

$$
\begin{aligned}
0= & \left(\left(\bar{Y}+\lambda^{y} \theta_{y}\right)^{2}+\sigma_{Y}^{2}\left(\eta_{1}^{*}\right)^{2}-2 \rho \sigma_{Y}\left(\bar{Y}+\lambda^{y} \theta_{y}\right) \eta_{1}^{*}\right) \phi^{2} \\
& +\left(2 \rho \sigma_{Y}\left(\bar{Y}+\lambda^{y} \theta_{y}\right) \eta_{0}+2 \kappa_{Y}\left(\bar{Y}+\lambda^{y} \theta_{y}\right) \eta_{1}^{*}-\left(\bar{Y}+\lambda^{y} \theta_{y}\right)^{2}-2 \sigma_{Y}^{2} \eta_{0} \eta_{1}^{*}\right) \phi \\
& +\left(\sigma_{Y}^{2} \eta_{0}^{2}-2 \kappa_{Y}\left(\bar{Y}+\lambda^{y} \theta_{y}\right) \eta_{0}\right) .
\end{aligned}
$$

Parameters that satisfy the relationship $\eta_{0}<2 \kappa_{Y}\left(\bar{Y}+\lambda^{y} \theta_{y}\right) / \sigma_{Y}^{2}$ guarantee that one root $\left(\phi_{1}\right)$ is negative for puts, whereas the other $\left(\phi_{2}\right)$ is positive for calls. The successive derivation is similar to those described for Equations (13) - (21). The characteristic function of this model is also provided in Appendix A. 


\section{NUMERICAL RESULTS AND COMPARISONS}

Longstaff and Schwartz (2001) proposed a simple least-squares method (LSM) to value American options by simulation. Table II gives a comparison of the LSM and the quadratic approximation proposed in this research for the stochastic volatility model with correlated double jumps. The parameters reported in Bakshi and Cao (2003) are used for this computation. The simulation is based on 20,000 paths for the stock-price process and the option is exercisable 20 times before maturity. Puts (P) and Calls $(\mathrm{C})$ are abbreviated as accordingly. The LSM proposed by Longstaff and Schwartz (2001), the quadratic approximation, and their difference, are represented by Simulated American, Approx, and Diff, respectively. The standard errors of the simulation estimates (s.e.) are given in parentheses. As shown, the differences between the LSM and the quadratic approximation (Approx) are typically small.

Table III gives a comparison of the LSM and the quadratic approximation for the stochastic volatility model with independent double jumps using parameters presented in Bakshi and Cao (2003). The differences between the LSM and the quadratic approximation presented in this research are also typically small. By turning off the specification of volatility jumps $\left(\lambda^{y}=0\right)$, the approximation formula reduces to the solution of the stochastic volatility model with return jumps empirically examined in Bakshi et al. (1997) and Bates (1996). As shown in Table IV, the differences between the LSM and the quadratic approximation are small. Table V exhibits that the differences between the LSM and

TABLE III

Comparisons of American Options: Independent Double Jumps

\begin{tabular}{|c|c|c|c|c|c|c|}
\hline & $S$ & European Option & Simulated American & (s.e.) & Approx & Diff \\
\hline \multirow[t]{7}{*}{$P$} & 60 & 39.423 & 39.999 & 0.013 & 40.000 & -0.001 \\
\hline & 70 & 29.749 & 30.065 & 0.028 & 30.090 & -0.025 \\
\hline & 80 & 20.747 & 20.899 & 0.040 & 20.950 & -0.050 \\
\hline & 90 & 13.261 & 13.328 & 0.046 & 13.389 & -0.060 \\
\hline & 100 & 7.843 & 7.908 & 0.070 & 7.927 & -0.019 \\
\hline & 110 & 4.381 & 4.426 & 0.047 & 4.439 & -0.013 \\
\hline & 120 & 2.361 & 2.396 & 0.035 & 2.403 & -0.007 \\
\hline \multirow[t]{7}{*}{ C } & 60 & 0.019 & 0.020 & 0.001 & 0.019 & 0.001 \\
\hline & 70 & 0.195 & 0.198 & 0.005 & 0.197 & 0.001 \\
\hline & 80 & 1.045 & 1.049 & 0.009 & 1.049 & 0.000 \\
\hline & 90 & 3.410 & 3.425 & 0.018 & 3.419 & 0.006 \\
\hline & 100 & 7.843 & 7.877 & 0.019 & 7.863 & 0.014 \\
\hline & 110 & 14.232 & 14.287 & 0.039 & 14.274 & 0.013 \\
\hline & 120 & 22.064 & 22.171 & 0.027 & 22.145 & 0.026 \\
\hline
\end{tabular}

Note. $K=100, T=0.25, r=0.06, d=0.06, \bar{Y}=0.49, Y=0.1623, \rho=-0.31, \lambda^{x}=0.87, \mu_{x}=-0.014, \sigma_{x}=0.04$, $\lambda^{y}=2.43, \theta_{y}=0.0036, \sigma_{Y}=0.54, \kappa_{Y}=3.02$, and $\alpha=1$. 
TABLE IV

Comparisons of American Options: Stochastic Volatility With Jump in Return

\begin{tabular}{|c|c|c|c|c|c|c|}
\hline & $S$ & European Option & Simulated American & (s.e.) & Approx & Diff \\
\hline \multirow[t]{7}{*}{$P$} & 60 & 39.556 & 39.998 & 0.030 & 40.000 & -0.002 \\
\hline & 70 & 30.156 & 30.400 & 0.046 & 30.309 & 0.091 \\
\hline & 80 & 21.564 & 21.636 & 0.084 & 21.623 & 0.013 \\
\hline & 90 & 14.436 & 14.505 & 0.044 & 14.461 & 0.043 \\
\hline & 100 & 9.181 & 9.247 & 0.061 & 9.193 & 0.054 \\
\hline & 110 & 5.686 & 5.722 & 0.048 & 5.692 & 0.030 \\
\hline & 120 & 3.505 & 3.519 & 0.046 & 3.508 & 0.011 \\
\hline \multirow[t]{7}{*}{ C } & 60 & 0.151 & 0.166 & 0.009 & 0.152 & 0.014 \\
\hline & 70 & 0.603 & 0.628 & 0.027 & 0.605 & 0.024 \\
\hline & 80 & 1.862 & 1.893 & 0.038 & 1.866 & 0.027 \\
\hline & 90 & 4.585 & 4.660 & 0.082 & 4.594 & 0.066 \\
\hline & 100 & 9.181 & 9.206 & 0.109 & 9.200 & 0.006 \\
\hline & 110 & 15.537 & 15.611 & 0.063 & 15.572 & 0.039 \\
\hline & 120 & 23.207 & 23.350 & 0.079 & 23.271 & 0.079 \\
\hline
\end{tabular}

Note. $K=100, T=0.25, r=0.06, d=0.06, \bar{Y}=0.49, Y=0.125, \rho=-0.16, \lambda^{x}=3.05, \mu_{x}=-0.03, \sigma_{x}=0.19, \lambda^{y}=0$, $\theta_{y}=0, \sigma_{Y}=0.41, \kappa_{Y}=3.92$, and $\alpha=1$.

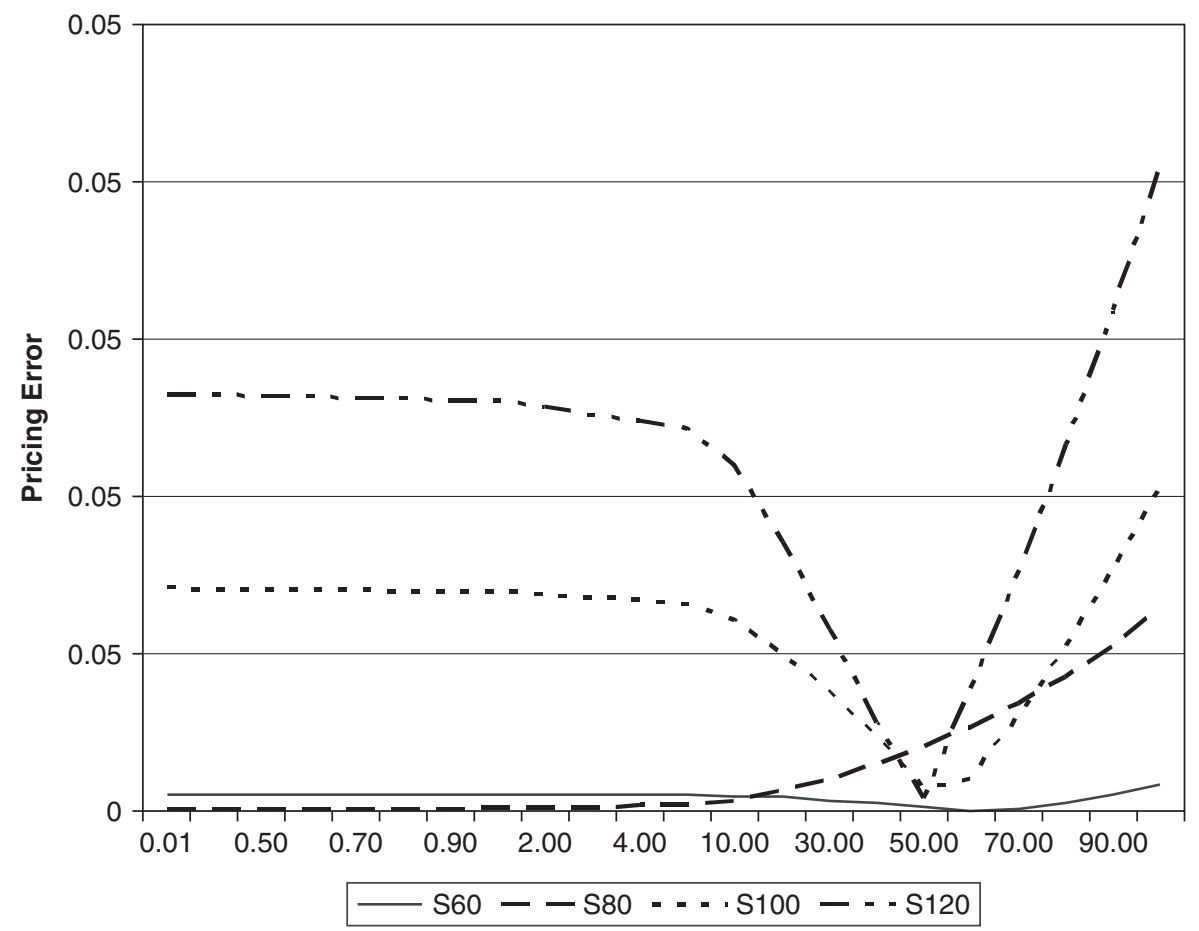

FIGURE 1

The choice of $\alpha$ in reducing pricing errors for calls. Note: $K=100, T=0.25, r=0.06$, $d=0.06, \bar{Y}=0.49, Y=0.1623, \rho=-0.31, \lambda^{x}=0.87, \mu_{x}=-0.014, \sigma_{x}=0.04, \lambda^{y}=2.43$, $\theta_{y}=0.0036, \sigma_{Y}=0.54$, and $\kappa_{Y}=3.02$. S60, S80, S 100 , and S 120 denote cases for which $S=60, S=80, S=100$, and $S=120$. 


\section{TABLE V}

Comparisons of American Options: Stochastic Volatility With Jump in Volatility

\begin{tabular}{|c|c|c|c|c|c|c|}
\hline & $S$ & European Option & Simulated American & (s.e.) & Approx & Diff \\
\hline \multirow[t]{7}{*}{$\mathrm{P}$} & 60 & 39.445 & 40.012 & 0.032 & 40.000 & 0.012 \\
\hline & 70 & 29.865 & 30.188 & 0.054 & 30.194 & -0.006 \\
\hline & 80 & 21.066 & 21.245 & 0.053 & 21.273 & -0.028 \\
\hline & 90 & 13.783 & 13.860 & 0.068 & 13.920 & -0.061 \\
\hline & 100 & 8.439 & 8.488 & 0.063 & 8.534 & -0.046 \\
\hline & 110 & 4.915 & 4.964 & 0.040 & 4.983 & -0.019 \\
\hline & 120 & 2.770 & 2.778 & 0.044 & 2.821 & -0.043 \\
\hline \multirow[t]{7}{*}{ C } & 60 & 0.040 & 0.046 & 0.006 & 0.041 & 0.005 \\
\hline & 70 & 0.311 & 0.325 & 0.021 & 0.314 & 0.011 \\
\hline & 80 & 1.363 & 1.402 & 0.031 & 1.369 & 0.033 \\
\hline & 90 & 3.932 & 3.983 & 0.053 & 3.945 & 0.038 \\
\hline & 100 & 8.439 & 8.498 & 0.056 & 8.466 & 0.032 \\
\hline & 110 & 14.766 & 14.882 & 0.067 & 14.817 & 0.064 \\
\hline & 120 & 22.472 & 22.652 & 0.104 & 22.564 & 0.088 \\
\hline
\end{tabular}

Note. $K=100, T=0.25, r=0.06, d=0.06, \bar{Y}=0.49, Y=0.189922, \rho=-0.26, \lambda^{y}=0, \mu_{x}=0, \sigma_{x}=0, \lambda^{y}=1.36$, $\theta_{y}=0.0016, \sigma_{Y}=0.53, \kappa_{Y}=2.58$, and $\alpha=1$.

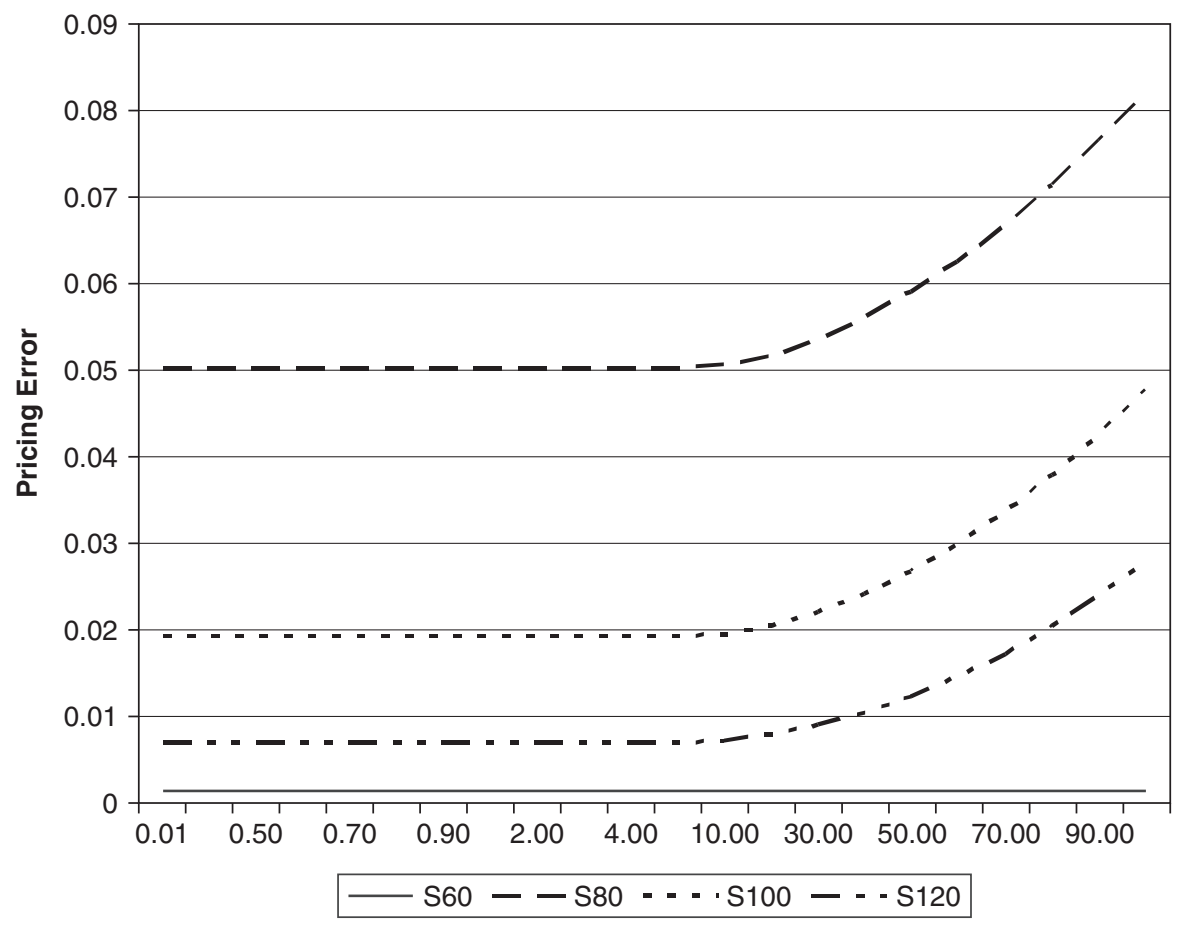

FIGURE 2

The choice of $\alpha$ in reducing pricing errors for puts. Note: $K=100, T=0.25, r=0.06$, $d=0.06, \bar{Y}=0.49, Y=0.1623, \rho=-0.31, \lambda^{x}=0.87, \mu_{x}=-0.014, \sigma_{x}=0.04, \lambda^{y}=2.43$, $\theta_{y}=0.0036, \sigma_{Y}=0.54$, and $\kappa_{Y}=3.02 . \mathrm{S} 60, \mathrm{~S} 80, \mathrm{~S} 100$, and $\mathrm{S} 120$ denote cases for which $S=60, S=80, S=100$, and $S=120$. 
the authors' approximation for the stochastic volatility model with volatility jumps $\left(\lambda^{x}=0\right)$ are small. These computations are performed with the parameters referenced from Bakshi and Cao (2003).

Figures 1 and 2 illustrate that the choice of $\alpha$ affects the pricing errors of the quadratic approximation for the stochastic volatility model with independent double jumps. Although the optimal choice of $\alpha$, between 50 and 60, minimizes pricing errors for calls, a corresponding choice for puts in this case cannot be determined. Therefore, the approach of Barone-Adesi and Whaley (1987) is followed and a value of $\alpha=1$ is selected for simplicity in these cases because the pricing errors are small and often well within the market bid-ask spread.

\section{CONCLUSIONS}

In this article, the application of a quadratic approximation method is described to obtain efficient analytic formulae for American options on processes permitting stochastic volatility and double jumps to illustrate its generality. To the best of one's knowledge, the literature suggests no approximation formulae for American option values based on these processes. This quadratic approximation scheme is a generalization of the Barone-Adesi and Whaley approach and nests several option models.

The constant volatility model is the first special case. The solution, with parameters specified as $\sigma_{Y}=0, \kappa_{Y} Y=\bar{Y}, \lambda^{y}=0$, and $\lambda^{x}=0$, reduces to that of Barone and Whaley in 1987. Bates' constant volatility model with return jumps presented in 1991 is the second special case $\left(\sigma_{Y}=0, \kappa_{Y} Y=\bar{Y}, \lambda^{y}=0\right.$, and $\lambda^{x}$ $\neq 0$ ). The third special case is Heston's (1993) stochastic volatility model without jumps ( $\lambda^{x}=0$ and $\left.\lambda^{y}=0\right)$ proposed in 1993. The stochastic volatility model, with return jumps, empirically examined in Bakshi et al. (1997) and Bates (1996) $\left(\lambda^{y}=0\right)$, is the fourth special case. The fifth special case is the stochastic volatility model with volatility jumps $\left(\lambda^{x}=0\right)$ proposed by Duffie et al.(2000). Moreover, the stochastic volatility model with correlated double jumps is also included in this scheme.

Comparisons with the least-squares approach show that the quadratic approximation scheme is very useful and efficient in pricing American options with stochastic volatility and double jumps. It is anticipated that the scheme can be further applied to American exotic options in the future. 


\section{APPENDIX A}

\section{Stochastic Volatility Model With \\ Correlated Double Jumps}

The present value of a basic European call option can be formulated as $C^{E}(S, Y, T ; K)=E^{\mathcal{Q}}\left[e^{-r T} \max \{S(T)-K, 0\}\right]$ and is given by

$$
\begin{aligned}
C^{E}(S, Y, T ; K)= & \frac{1}{2} J(T ;-i)-\frac{1}{\pi} \int_{0}^{\infty} \frac{\operatorname{Im}[J(T ;-i-\nu) \exp [i \nu \log [K]]]}{\nu} d \nu \\
& -K\left(\frac{1}{2} J(T ; 0)-\frac{1}{\pi} \int_{0}^{\infty} \frac{\operatorname{Im}[J(T ;-\nu) \exp [i \nu \log [K]]]}{\nu} d \nu\right.
\end{aligned}
$$

where $J(T ; \phi)$ is the characteristic function of the state density. The characteristic function is given by

$$
J(T ; \phi)=\exp [A(T ; \phi)+B(T ; \phi) Y] S^{i \phi}
$$

where $A(T ; \phi)$ and $B(T ; \phi)$ are

$$
\begin{aligned}
& A(T ; \phi) \equiv(i \phi(r-d)-r) T \\
& -\frac{\bar{Y}}{\sigma_{Y}^{2}}\left[\left(\varepsilon+i \phi \sigma_{Y} \rho-\kappa_{Y}\right) T+2 \log \left[1-\frac{\left(\varepsilon+i \phi \sigma_{Y} \rho-\kappa_{Y}\right)(1-\exp [-\varepsilon T])}{2 \varepsilon}\right]\right] \\
& -i \phi \lambda^{x, y}\left(\frac{\exp \left(\mu_{0}+\frac{1}{2} \sigma_{x, y}^{2}\right)}{1-\theta_{y} \mu_{x, y}}-1\right) T-\lambda^{x, y} T \\
& +\frac{\lambda^{x, y}(2 \varepsilon-b) \exp \left(i \phi \mu_{0}-\frac{1}{2} \phi^{2} \sigma_{x, y}^{2}\right) T}{p} \\
& +\frac{2 \lambda^{x, y} \theta_{y} i \phi(i \phi-1) \exp \left(i \phi \mu_{0}-\frac{1}{2} \phi^{2} \sigma_{x, y}^{2}\right)}{p q} \log \left(\frac{p+q e^{-\varepsilon T}}{p+q}\right) \\
& B(T ; \phi) \equiv \frac{i \phi(i \phi-1)(1-\exp [-\varepsilon T])}{2 \varepsilon-\left(\varepsilon+i \phi \sigma_{Y} \rho-\kappa_{Y}\right)(1-\exp [\varepsilon T])} \\
& \varepsilon \equiv \sqrt{\left(i \phi \sigma_{Y} \rho-\kappa_{Y}\right)^{2}-i \phi(i \phi-1) \sigma_{Y}^{2}}
\end{aligned}
$$




$$
\begin{aligned}
b & \equiv \varepsilon+i \phi \sigma_{Y} \rho-\kappa_{Y} \\
p & \equiv 2 \varepsilon\left(1-\theta_{y} i \phi \mu_{x, y}\right)-q \\
q & \equiv i \phi(i \phi-1) \theta_{y}+b\left(1-\theta_{y} i \phi \mu_{x, y}\right) .
\end{aligned}
$$

The proof is published in Duffie et al. (2000). Given the solution for European calls, the formula for puts, $P^{E}(S, Y, T ; K)$, can be obtained by the put-to-call conversion equation (Grabbe, 1983):

$$
P^{E}(S, Y, T ; K)=C^{E}(S, Y, T ; K)-S \exp [-d T]+K \exp [-r T] .
$$

\section{Stochastic Volatility Model With Independent Double Jumps}

The characteristic function of the state density has the same functional form as in Equation (A2). However, the component function $A(T ; \phi)$ is somewhat different than Equation (A3).

$$
\begin{aligned}
& A(T ; \phi) \equiv(i \phi(r-d)-r) T-i \phi \lambda^{x} \mu_{x} T \\
&+\lambda^{x}\left[\left(1+\mu_{x}\right)^{i \phi} \exp \left[\frac{1}{2} i \phi(i \phi-1) \sigma_{x}^{2}\right]-1\right] T \\
&-\frac{\bar{Y}}{\sigma_{Y}^{2}}\left[\left(\varepsilon+i \phi \sigma_{Y} \rho-\kappa_{Y}\right) T+2 \log \left[1-\frac{\left(\varepsilon+i \phi \sigma_{Y} \rho-\kappa_{Y}\right)(1-\exp [-\varepsilon T])}{2 \varepsilon}\right]\right] \\
&-\lambda^{y} T+\frac{\lambda^{y}(2 \varepsilon-b)}{2 \varepsilon-q} T+\frac{2 \lambda^{y}(q-b)}{(2 \varepsilon-q) q} \ln \left[\frac{2 \varepsilon-q(1-\exp [-\varepsilon T])}{2 \varepsilon}\right] \\
& q \equiv b+i \phi(i \phi-1) \theta_{y} .
\end{aligned}
$$

\section{BIBLIOGRAPHY}

Bakshi, G., \& Cao, C. (2003). Risk-neutral kurtosis, jumps, and option pricing: Evidence from 100 most actively traded firms on the CBOE. EFA 2003 Annual Conference Paper No. 953.

Bakshi, G., Cao, C., \& Chen, Z. (1997). Empirical performance of alternative option pricing models. Journal of Finance, 53, 499-547.

Barone-Adesi, G., \& Whaley, R. (1987). Efficient analytic approximation of American option values. Journal of Finance, 42, 301-320.

Bates, D. (1991). The crash of ' 87 : Was it expected? The evidence from options markets. Journal of Finance, 46, 69-107.

Bates, D. (1996). Jumps and stochastic volatility: exchange rate processes implicit in PHLX deutsche mark options. Review of Financial Studies, 9, 69-107. 
Broadie, M., Chernov, M., \& Johannes, M. (2007). Model specification and risk premiums: The evidence from the futures options. Journal of Finance, 62, 1453-1490.

Chang, G., Kang, J., Kim, H., \& Kim, I. (2007). An efficient approximation method for American exotic options. Journal of Futures Markets, 27, 1, 2007.

Duffie, D., Pan, J., \& Singleton, K. (2000). Transform analysis and asset pricing for affine jump-duffusions. Econometrica, 68, 6, 1343-1376.

Grabbe, J. (1983). The pricing of call and put options on foreign exchange. Journal of International Money and Finance, 2, 239-253.

Guo, J., \& Hung, M. (2007). A note on the discontinuity problem in Heston's stochastic volatility model. Applied Mathematical Finance, 14, 339-345.

Heston, S. (1993). A closed form solution for options with stochastic volatility with applications to bond and currency options. Review of Financial Studies, 6, $327-343$.

Ju, N., \& Zhong, R. (1999). An approximate formula for pricing American options. Journal of Derivatives, 7, 31-40.

Longstaff, F., \& Schwartz, E. (2001). Valuing American options by simulation: A simple least-squares approach. Review of Financial Studies, 14, 113-147.

MacMillan, L. (1987). Analytic approximation for the American put option. Futures and Options Research, 1A, 119-139. 